\title{
Attribute Reduction Algorithm Based Early Warning Model of Sports Injury
}

\author{
Xiaofei Wang and Yonghong Guo \\ Lishui University, Faculty of Education Lishui, Zhejiang, 323000, China \\ E-mail: Xiaofeiwang1010@outlook.com, Yonghongguo221@outlook.com
}

Keywords: attribute reduction algorithm, sports injury, early warning model, psychological training, medical supervision

Received: May 21, 2021

\begin{abstract}
With the continuous development of track and field sports, a relatively complete and scientific system has been formed in terms of training, and more attention has been paid to the planned training of teenagers for many years, psychological training and recovery training, and strengthening medical supervision and scientific research. In order to speed up the scientific training of track and field and improve the level of track and field training in China, in view of the characteristics of track and field injuries such as multiple causes and complexity, this paper analyzes and studies the attribute reduction algorithm based on attribute reduction algorithm, and proposes a sports injury early warning model based on mutual information. Taking the reduction results as input neurons, a BP neural network with hidden layer is established in MATLAB environment. The results show that: call $85 \%$ of the track and field athletes in the sample information as training samples for training. After the simulation experiment of the model, it is found that the error value of the prediction results is basically controlled in the range of-0.025-0.05, which meets the requirements of early warning accuracy of injury risk level of athletes, and the accuracy rate of early warning of sports injury risk reaches $100 \%$.
\end{abstract}

Povzetek: Razvit je sistem za zgodnje napovedovanje športnih poškodb.

\section{Introduction}

Track and field is one of the important sports events. It includes race walking, middle and long distance running, jumping, throwing and all-round sports, with more than 40 single events. It is one of the important means to enhance people's physical fitness and the foundation of various sports. With the continuous development of track and field sports, a relatively complete and scientific system has been formed in terms of training, and more attention has been paid to the planned training of teenagers for many years, psychological training and recovery training, and strengthening medical supervision and scientific research $[1,2]$.The improvement of field equipment, and many scientific research results directly or indirectly applied to track and field sports practice, doping detection means also reached a very high level, all of which promote and ensure the healthy and rapid development of track and field and the improvement of sports performance. In order to obtain good results, athletes have to pay more efforts [3]. For example, China's 110 meter hurdles athlete Liu Xiang left the Olympic Games in Beijing in 200 years because of inflammation of his Achilles tendon. In June 2012, Liu Xiangping, a flying man in the Youjin station of the Diamond League, won the world record and won the championship. However, he withdrew from the Olympic Games due to injury before the final in London in midJuly. At the London Olympic Games in August 2012, Liu Xiang left the Olympic Games with doubts and praises because of his Achilles tendon injury $[4,5]$.Therefore, it is of direct and important significance to investigate and analyze the injuries and injuries of professional athletes in track and field, summarize their injuries, similarities in similar events, injury prone time periods and parts, and reduce the incidence of sports injuries, which is of direct and important significance for ensuring athletes' sports skills and career. It is urgent to find out the cause of sports injury and use more scientific means or methods to

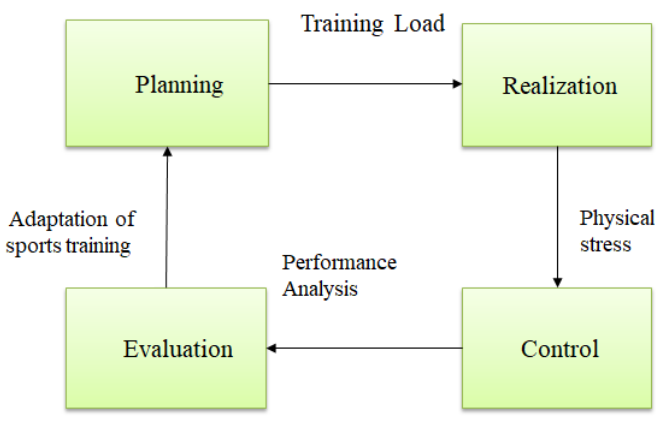

Figure 1: Systematic review of the system.

prevent injury $[6,7]$. The systematic review of the system is shown in Figure 1.

In the professional sports, muscle injuries at the lower extremity are very common like handball, soccer and rugby. The sudden acceleration and deceleration tasks are required by the sports with the direction change. There are many situations in which a ball is repetitively kicked by 
the players. A typical professional soccer team is demonstrated by the data with 25-player squad and more than a quarter can be counted from injuries $[8,9]$. The more than $90 \%$ of all Lower extremity muscle injuries (MUSINJ) in soccer is comprised by the lower extremity of four major muscle groups' injuries. The development and implementation of strategies are required that prevent and reduce the MUSINJ severity. The identification of athletes at high risk of MUSINJ is done for screening program validation for MUSINJ prevention program establishment [10]. For prediction and prevention of sports injuries, the three steps are overcome successfully.

Identification of potential risk factors to demonstrate the strong relationship with injury in prospective studies with the cut-off values.

The validity of the screening tests are determined that are utilized to measure the risk factors for new athlete population prediction.

Identification of the intervention programme to target the athletes at high risk by utilizing the developed screen.

The organization of the paper is as follows. Section II provides an overview of the exhaustive literature survey followed by a methodology adopted in section III. A detailed discussion of obtained results is in section IV. Finally, Section V concludes the paper.

\section{Literature review}

For track and field injuries, researchers at home and abroad have made a lot of research. Starting from the training practice, Shao Liwei proposed to take the prevention of injury as an important part of training arrangement. After injury, active recovery as an important auxiliary treatment can achieve good results. It is emphasized that the combination of coaches and doctors can improve the prevention level of thigh muscle injury of Female Sprinters. Liu Ju made a follow-up investigation and Analysis on the thigh muscle injury in hurdles, put forward the prevention measures of stress, and adopted the method of electronic conduction therapy instrument to reduce the occurrence of thigh muscle injury in hurdle, ensure training and competition, improve the level of sports, and promote the development of track and field [11]. Nikolaos evaluated 165 track and field athletes with acute posterior femoral muscle injury using the same nonsurgical rehabilitation program. In the research sample, $81 \%$ of the affected limb's range of motion is 20 degrees smaller, the recovery time is 2 weeks, the range of motion is more than 30 degrees accounted for $3.6 \%$, recovery time more than 6 weeks, complete recovery time and range of motion defects have obvious relationship, the measurement of the range of motion of knee joint defects is conducive to predict the recovery time of athletes. In order to vividly describe the relationship between internal risk factors, external risk factors, stimulus events and sports injury in the process of sports training, established a dynamic etiological model of sports injury. In the model, the internal risk factors such as age, body composition, physical fitness, injury history, abnormal anatomical structure are described; external factors include protective measures, equipment and facilities, environmental factors, etc.; stimulating or inducing events include joint activity, training plan, competition schedule, etc [12]. He believes that internal risk factors will not directly lead to injury, only have the tendency of injury. If combined with the role of external risk factors, it is likely to make athletes become vulnerable to injury. If there is stimulation or induced event at this time, it will lead to injury. He also stressed that attention should be paid to the interaction between internal and external risk factors. Attribute reduction algorithm is the core content of rough set theory [13]. After years of research and development, scholars at home and abroad have developed many kinds of attribute reduction algorithms. Scholars from different angles, using a variety of technologies and strategies to study, and thus put forward a lot of algorithms, mainly based on discernibility matrix attribute reduction algorithm, information entropy, knowledge granularity based heuristic attribute reduction algorithm, based on positive domain and dependency degree of attribute reduction algorithm and so on [14]. As a new mathematical tool, the advantage of rough set theory is that it can discover the inner relation or decision rules of problems through knowledge reduction based on the classification ability of data, without any prior knowledge and additional information of data. The improvement of existing algorithms and the development of small-scale systems are also a very valuable research direction [15, 16]. Especially in the sports field, there is a lack of reduction algorithm based on the characteristics of sports events. The provide reference for the treatment and prevention of sports injury is provided [17].

The earthquake is detected by the Earthquake Early Warning (EEW) systems and strong shaking intensity notice is provided [18]. On society, EEW's potential impacts are understood with the advancement in the EEWs technical research. Benefits are provided by the EEW perceived by the participants like life-safety and health \& safety, psychological preparedness, activation of emergency plans and situational assessment and organizational. There is rapid development of EEW technological research that is useful sectorial applications. The CET 4 affected by the many factors are analyzed based on the multiple linear regression algorithms [19]. The statistical analysis techniques are utilized for transformation of history data based on the data mining and then CET- 4 test result and its factors are obtained. There is high relationship between the degrees of fit. The partition-weighted K-nearest neighbor algorithm is established to further improve the algorithm. There is no significant improvement but the stability classification is better than K-nearest neighbor algorithm, its classification efficiency is greatly improved, classification time is greatly reduced, and classification efficiency is increased by $19 \%$. The individual's heat-stress response is continually learned by the environmental variables and the individual's noninvasive measurements are utilized by the model [20]. The real-time individualized core body temperature estimate is provided by the model parameters. In this paper, author details the contemporary statistical approaches from Machine Learning and Data Mining environments for robust predictive models building [21]. 
The numerous machines learning behavior is analyzed and compared lower extremity muscle injuries risk identification. All the MUSINJ occurring in the 2013/2014 seasons are captured by employing the injury surveillance. The analysis and comparison of predictive ability of several models are done by applying a range of learning techniques.

Main focus of this work is to speed up the scientific training of track and field and improve the level of track and field training in China, in view of the characteristics of track and field injuries such as multiple causes and complexity.

1. The attribute reduction algorithm based on attribute reduction algorithm is analysed properly.

2. The sports injury early warning model is proposed which is based on the mutual information.

3. Establishment of BP neural network with hidden layer in the MATLAB by reduction results as input neurons.

\section{The realization of attribute reduction algorithm}

\subsection{Attribute reduction based on mutual information}

Attribute reduction based on mutual information is to express the basic concept and operation of rough sugar set from the perspective of information theory. The basic information of mutual information has been introduced in the previous $[22,23]$. The basic idea is to select the most important attribute set to add to the core based on the core of the condition attribute relative to the decision attribute in the decision table, and finally take the mutual information equality as the end condition of reduction.

Algorithm description:

Input: Information System, $\mathrm{S}=(\mathrm{U}, \mathrm{A}, \mathrm{V}, \mathrm{F})$. In which, $\mathrm{A}=\mathrm{C} \cup \mathrm{D}, \mathrm{C} \cup \mathrm{D}=\phi$ 。

Where, $U$ is the universe, $\mathrm{C}$ and $D$ are the condition and decision attribute set respectively.

Output: Relative reduction of C to D.

1) The mutual information $I(C ; D)$ of condition attribute $\mathrm{C}$ relative to decision attribute $\mathrm{d}$ is calculated;

$\mathrm{D}, \mathrm{I}(\mathrm{R} ; \mathrm{D})<\mathrm{I}(\mathrm{C} ; \mathrm{D})$; Sometimes, relative kerne is $\mathrm{R}=\phi \mathrm{I}(R ; D)=0$.

2) Let $B=R$ and the condition attribute $\mathrm{C}$-B repeat: Any attribute $\mathrm{P} \in \mathrm{C}-\mathrm{B}$ computing mutual information $\mathrm{I}(p ; D \mid B)$; extract the attribute that maximizes the conditional mutual information $I(p ; D \mid \mathrm{b})$, if there are multiple attributes $\mathrm{p}_{i}(i=$ $1,2, \ldots, n)$ when the attribute reaches the maximum value, the attribute $P i$ with the least number of attribute value combinations with $\mathrm{B}$ is selected.

3) Order $\mathrm{B}=\mathrm{B} \cup\left(a_{i}\right)$;

4) If $I(B ; d)=I(C ; D)$, terminate; otherwise, turn to step 3.

5) Finally, $B$ is a relative reduction relative to $C$.

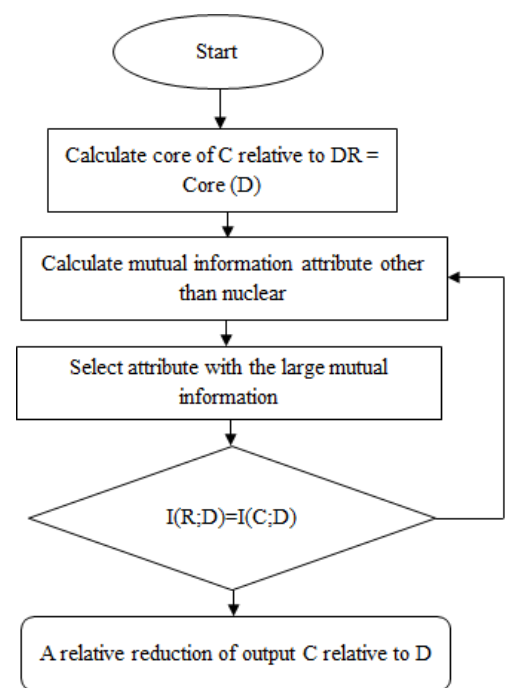

Figure 2: Flow chart of attribute reduction algorithm based on mutual information.

The flow chart of attribute reduction algorithm based on mutual information is shown in Figure 2.

Based on mutual information attribute reduction algorithm, an attribute importance reduction method is defined from the perspective of information [24, 25]. In the worst case, the number of attributes considered is $\mathrm{C}$, $C-11$.So the total reduction number is $m+m+1+$ $1=M(M+1) / 2$. If the influence of the number of objects on the calculation time is ignored, the algorithm can find a satisfactory reduction in $O(C 2)$ time complexity in the worst case [26].

\subsection{Sample and index selection}

In this paper, through questionnaire survey and expert interview to summarize the potential injury factors, and collect and process the data.

1) Establishment of data collection table of injury risk factors in track and field sports: After consulting a large number of domestic and foreign literatures and interviewing with experts in sports medicine, excellent teachers and athletes of track and field team, the incidence and common causes of sports injury in track and field events were understood. Based on the in-depth analysis and systematic classification of injury factors, the data collection table of injury risk factors in track and field sports was formulated [27-29]. It is used to collect the information of injury factors and injury database. The data collection form is distributed to athletes once a week in the training cycle, and the athletes fill in according to their own actual situation.

2) Data statistics of injury risk factors in track and field sports: Firstly, the factors related to sports injury risk in data collection table of injury risk factors of track and field sports" were screened out, and the corresponding options of the factors were assigned according to their contribution to sports injury risk [30]. Taking three option factors as an example, the minimum contribution of option level is assigned to 1 , and the maximum option is assigned to 3 . On this basis, the standard form is used to make statistics on the data collection form filled in by athletes. 


\subsection{Injury surveillance}

The International Injury Consensus Group make the recommendation, an acute pain occurred during training or competition in the muscle is the MUSINJ. The immediate termination of play is resulted in the next training session. A clinical examination is done to confirm the injuries by the doctors. The full participation in training is allowed by the players who are considered injured and the match selection availability is also allowed. There are different kinds of studies and in this study, triceps surae and adductor muscles injuries are considered. At the club, there are medical staff recorded MUSINJ on an injury sent to the study group each month. The following details are provided by the medical staff to the investigators [31]. The muscle, leg injured, injury severity based on lay off time from soccer and whether it was a recurrence and total time for full training and competition resuming. After follow up period, the individual clubs data is collected into the central database that followed up at the different clubs. The minimal MUSINJ is diagnosed and the total time loss is recorded by the medical staff. In the in the injury surveillance process, these inconsistencies are resolved and only MUSINJ showing a time lost $>4$ days are then analyzed statistically. The testing procedure is graphically represented in Figure 3.

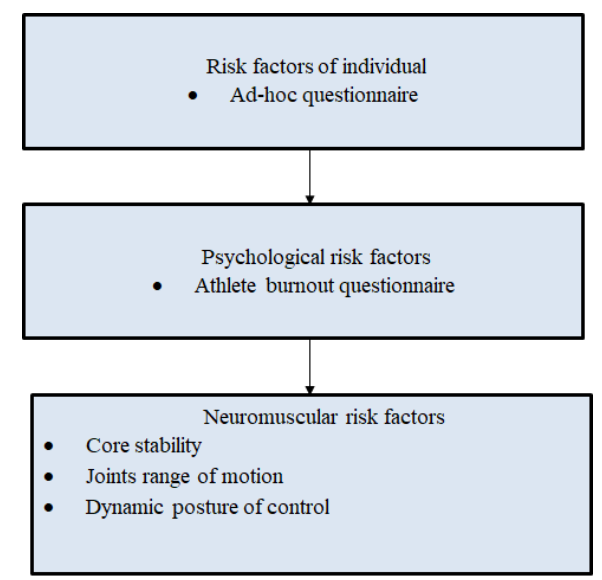

Figure 3: Graphically representation of testing procedure.

\subsection{Statistical analysis}

The several techniques are analyzed and compared by the statistical analysis to find the predicting MUSINJ model [32-34]. The two stages comprise the statistical analysis.

1) Data pre-processing: The data mining techniques are applied by preparing the datasets in this stage. The data cleaning and data discretization are the pre-processing methods applied for optimization.

2) Data processing: The imbalanced data sets are applied and address the learning at this stage. The pre-processing, cost-sensitive learning and ensemble-based methods are studied [35-37]. The combination of a cost-sensitive classifier with class-balanced ensembles for imbalanced data sets.

\section{Results and discussion}

This project adopts expert validity test method to analyze and evaluate the whole, structure and content of the questionnaire (using the evaluation scale of very perfect, relatively perfect, perfect foundation, not too perfect and imperfect). By distributing questionnaires to 17 experts who are engaged in track and field professional team coaches, the evaluation results of experts on the effectiveness of the questionnaire are shown in Table 1 and graphically presented in Figure 4 for better analysis and visualization. The results show that no experts think that the overall evaluation, structure evaluation and content evaluation of the questionnaire are not perfect or imperfect. It can be considered that the validity evaluation of the questionnaire can meet the requirements of the questionnaire design. Reliability test: the reliability of the questionnaire is tested by half reliability test, and the content consistency coefficient is tested by Spearman Brown formula. The reliability coefficient and recovery rate of the questionnaire meet the needs of data analysis.

\begin{tabular}{cccc}
\hline & $\begin{array}{c}\text { Very } \\
\text { perfect }\end{array}$ & $\begin{array}{c}\text { More } \\
\text { perfect }\end{array}$ & $\begin{array}{c}\text { Basic } \\
\text { perfect }\end{array}$ \\
\hline $\begin{array}{c}\text { Overall } \\
\text { evaluation }\end{array}$ & & 93 & 7 \\
$\begin{array}{c}\text { Structure } \\
\text { evaluation }\end{array}$ & 7 & 50 & 43 \\
$\begin{array}{c}\text { Content } \\
\text { evaluation }\end{array}$ & 64 & 36 \\
\hline
\end{tabular}

Table 1: Expert validity evaluation results.

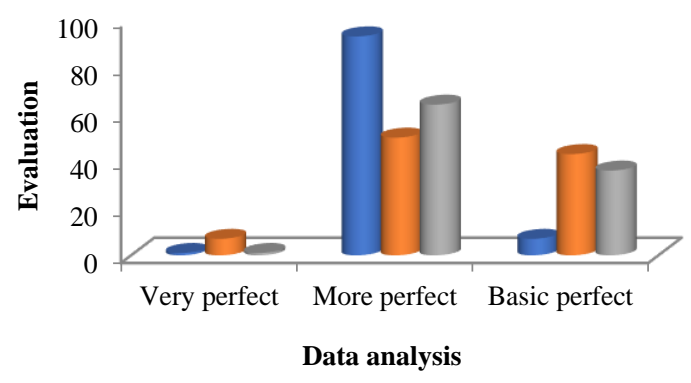

- Overall evaluation $\square$ Structure evaluation $\square$ Content evaluation

Figure 4: Expert validity evaluation results.

In order to comprehensively reflect the possible factors of injury, we will choose as many relevant indicators and data as possible when selecting injury indicators of athletes [38]. However, such a large number of indicators will not only affect the early warning effect of the model, but also cause the complexity of the system, which will limit the implementation of the transplantation of mobile platform. Therefore, this paper uses the powerful function of rough set theory in attribute reduction to select all the injury indicators and select the indexes with higher contribution rate to the next step. The first thing we need to do is to establish a knowledge expression system with conditional attributes and decision attributes, that is, the generation of decision table. 


\begin{tabular}{cccl}
\hline $\begin{array}{c}\text { Serial } \\
\text { number }\end{array}$ & $\begin{array}{c}\text { Model } \\
\text { warning } \\
\text { results }\end{array}$ & $\begin{array}{c}\text { The } \\
\text { actual } \\
\text { results }\end{array}$ & Risk level \\
\hline $\mathbf{1}$ & 1.04 & 1 & Low risk level \\
$\mathbf{2}$ & 2.02 & 2 & Low risk level \\
$\mathbf{3}$ & 3.03 & 3 & Low risk level \\
$\mathbf{4}$ & 2.98 & 3 & Low risk level \\
$\mathbf{5}$ & 2.02 & 2 & Low risk level \\
$\mathbf{6}$ & 2.00 & 1 & Low risk level \\
$\mathbf{7}$ & 0.95 & 1 & Low risk level \\
$\mathbf{8}$ & 2.00 & 2 & Low risk level \\
$\mathbf{9}$ & 2.01 & 2 & Low risk level \\
$\mathbf{1 0}$ & 1.01 & 1 & Low risk level \\
\hline
\end{tabular}

Table 3: Test results of sports injury early warning model (data in the table are adjusted to make the error between -0.025-0.05).

According to the collected injury information data of track and field athletes, the decision information table of injury factors and injury risk is established. In the table a, Band We are conditional attributes, which respectively represent the injury risk index of track and field injury; they are decision-making attributes and represent the risk level of sports injury.

\subsection{BP neural network training}

This paper mainly uses BP neural network, which is the most widely used neural network, to train the samples. According to the input information, the athletes can be divided into three categories according to the risk of sports injury. For simplicity, the three categories are represented by 1,2 , and 3 of BP neural network output value. Comparison table of BP output and risk level is tabulated in Table 2.

\begin{tabular}{cc}
\hline $\begin{array}{c}\text { BP neural network } \\
\text { output value }\end{array}$ & $\begin{array}{c}\text { Risk warning level of } \\
\text { sports injury }\end{array}$ \\
\hline $\mathbf{1}$ & Low risk level \\
$\mathbf{2}$ & Medium risk level \\
$\mathbf{3}$ & High risk level \\
\hline
\end{tabular}

Table 2: Comparison of BP output and risk level.

The statistical data of 80 track and field athletes were randomly divided into two groups. The statistical data of 70 athletes were taken as training samples and the rest 10 as test samples. BP network model was constructed in MATLAB environment.

The main parameters include the weight and threshold of each layer, the transfer function and training function of hidden layer and output layer, training step length, training times, training target precision, learning efficiency, etc. After repeated experiments and comparing the results of many experiments, the final conclusion is that when the number of neurons in hidden layer is 12 , the number of hidden layer neurons is 12 . When the training error is set to 0.001 , the learning efficiency is set to 0.03 , the training step size is 600 , and the maximum training times is 12000 , the prediction effect of reduction results based on mutual information is the best. When the error decreases to 0.001 , the curve gradually flattens, indicating that the structure achieves stability and good effect. However, the maximum number of attribute reduction results based on dependency degree reaches 22000 times, and the training error of the network is reduced to 0.006 , and the error curve tends to be gentle, which means that no matter how the BP network is trained, the error will not be greatly reduced.

\subsection{Sports injury early warning model test}

Using the neural network early warning model based on attribute reduction which has been established before, combined with the sports injury information of track and field athletes, the numerical value is input into the established model. Test results of sports injury early warning model is shown in Table 3.

The experimental results show that, using the trained neural network to test and verify 10 test samples, the more ideal results are obtained, and the error value of the prediction results is basically controlled between-0.0250.05 . Such results meet the requirements of early warning accuracy of athletes' injury risk level in this topic, and the accuracy rate of sports injury risk early warning reaches $100 \%$. Therefore, it is feasible to apply this algorithm to the early warning of sports injury risk of athletes. The combination of attribute reduction algorithm and neural network can not only improve the performance of the neural network, reduce the complexity of the network and reduce the training time of the network, but also effectively, conveniently and real-time prevent the occurrence of track and field sports injury to a certain extent. It has certain practical value in the actual training process.

\section{Conclusion}

In order to speed up the scientific track and field training and improve the level of track and field training in our country, aiming at the characteristics of track and field injuries such as multiple causes and complexity, this paper analyzes and studies the attribute reduction algorithm based on attribute importance, and proposes a reasonable and effective attribute reduction algorithm based on attribute importance, which is applied to the early warning model of track and field injury risk. This paper proposes an attribute reduction algorithm based on mutual information and realizes it with programming tools. Finally, the reduction result is obtained, which is the injury index set which has great influence on track and field athletes. Taking the reduction results as input neurons, a BP neural network with hidden layer is established in MATLAB environment, and $85 \%$ of the track and field athletes' sample information is used as training samples for training. The optimal model structure is determined by comparing the convergence and result error. Taking the rest of the samples of track and field athletes as test data, the simulation experiment was carried out on the model. The results showed that the error value of the prediction results was basically controlled between-0.025-0.05, which met the requirements of early warning accuracy of 
athletes' injury risk level, and the accuracy rate of sports injury risk early warning reached $100 \%$.

\section{Acknowledgement}

Research Center of Shangluo Culture and Jia Pingwa, A Study on the Translation and Introduction of Mo Yan's Works and Jia Pingwa's Works in English-speaking Countries (18SLWH05).

\section{References}

[1] Leihua, Z. , Hongtai, N. , Zhongni, W. , \& Xuehong, L. . (2019). Research on the construction of early warning model of criminals based on big data. Netinfo Security. https://doi.org/10.1007/978-981-32-9563-6_27

[2] Zhou, D. . (2019). Research on the security early warning model of campus network based on log. IOP Conference Series Earth and Environmental Science, 252, 042080 . doi:10.1088/1755-1315/252/4/042080

[3] Juan, D. , Yue, W. , Wei, J. , \& Shuyuan, L. . (2019). Research on the Early-warning Model of Foaming Phenomenon in Desulfurization Solution System of Natural Gas Purification Plant Based on Artificial Intelligence Technology. 2019 2nd International Conference on Artificial Intelligence and Big Data (ICAIBD). https://doi.org/10.1016/j.desal

[4] Jin, R. , \& Rui, C. . (2019). Research on early warning model of microblog public opinion based on netizens' interest preferences. China Public Security(Academy Edition). doi:10.1088/1742-6596/1631/1/012108

[5] Yang, P. , \& Yang, M. . (2019). Research on the Management Model of University Students Academic Early Warning Based on Big Data Analysis. International Conference on Communications, Information System and Computer Engineering. North China University of Technology;Beijing University of Chemical Technology.

DOI: 10.1109/CISCE.2019.00148

[6] Yu, C. , Li, Z. , \& Liu, H. . (2019). Research on Gravity Compensation Algorithm of Model-free Manipulator Based on L1-norm Convex Optimization. 2018 IEEE International Conference on Robotics and Biomimetics (ROBIO). IEEE. DOI: $10.1109 /$ ROBIO.2018.8664862

[7] Wang, X., (2020). Research on inversion of ecosystem dynamics model parameters based on improved neural network algorithm. Microprocessors and Microsystems, 80. DOI: 10.1016/j.micpro.2020.103605

[8] Jiang, H., Xue, Z. P. , \& Guo, Y. . (2020). Research on plant leaf disease identification based on transfer learning algorithm. Journal of Physics Conference Series, 1576, 012023. https://doi.org/10.1155/2020/2479172

[9] Qi, Z. , Li, S. , Li, M. , Dang, C. , Sun, D. , \& Zhang, D. , et al. (2019). Research on the algorithm model for measuring ocean waves based on satellite gps signals in china. Sensors (Basel, Switzerland), 19(3). https://doi.org/10.3390/s19030541

[10] Xin, C. , Dan, L. , \& Shuo, H. . (2020). Research on Deceleration Early Warning Model Based on V2X. 2020 12th International Conference on Measuring Technology and Mechatronics Automation (ICMTMA). https://doi.org/10.1155/2020/5172305

[11] Jia, X., \& Zhang, D. (2021). Prediction of maritime logistics service risks applying soft set based association rule: An early warning model. Reliability Engineering \& System Safety, 207, 107339. DOI: $10.1016 /$ j.ress.2020.107339

[12] Lowe, D., Ebi, K. L., \& Forsberg, B. (2011). Heatwave early warning systems and adaptation advice to reduce human health consequences of heatwaves. International journal of environmental research and public health, 8(12), 4623-4648. doi: 10.3390/ijerph8124623

[13] Delibaic, B., \& Obradovic, Z. (2012, April). Towards a DGSS prototype for early warning for ski injuries. In 2012 IEEE 28th International Conference on Data Engineering Workshops (pp. 68-72). IEEE. DOI: 10.1109/ICDEW.2012.73

[14] Ahmed, B., Rahman, M. S., Sammonds, P., Islam, R., \& Uddin, K. (2020). Application of geospatial technologies in developing a dynamic landslide early warning system in a humanitarian context: the Rohingya refugee crisis in Cox's Bazar, Bangladesh. Geomatics, Natural Hazards and Risk, 11(1), 446-468. doi/full/10.1080/19475705.2020.1730988

[15] Zhang, K., Zheng, X., Li, H., \& Zhao, Z. (2020). Human health risk assessment and early warning of heavy metal pollution in soil of a coal chemical Plant in Northwest China. Soil and Sediment Contamination: An International Journal, 29(5), 481-502. DOI: 10.1080/15320383.2020.1746737

[16] Fu, Z., Zhang, H., Chen, Q., \& Liu, S. (2016, December). Application of naive bayes classifier in stampede risk early-warning of large-scale activities In 2016 International Conference on Industrial Informatics-Computing Technology, Intelligent Technology, Industrial Information Integration (ICIICII) (pp. 174-180). IEEE.

DOI:10.1371/journal.pone.0150630

[17] Fu, Z., Zhang, H., Chen, Q., \& Liu, S. (2016, December). Application of naive bayes classifier in stampede risk early-warning of large-scale activities. In 2016 International Conference on Industrial Informatics-Computing Technology, Intelligent Technology, Industrial Information Integration (ICIICII) (pp. 174-180). IEEE. doi: 10.21037/atm.2016.03.38

[18] Becker, J. S., Potter, S. H., Prasanna, R., Tan, M. L., Payne, B. A., Holden, C., ... \& Johnston, D. M. (2020). Scoping the potential for Earthquake Early Warning in Aotearoa New Zealand: a sectoral analysis of perceived benefits and challenges. 
International Journal of Disaster Risk Reduction, 51, 101765 .

https://doi.org/10.5194/nhess-2021-42

[19] Xu, J., \& Liu, Y. (2019). CET-4 score analysis based on data mining technology. Cluster Computing, 22(2), 3583-3593. https://doi.org/10.1007/s10586-018-2208-x

[20] Laxminarayan, S., Rakesh, V., Oyama, T., Kazman, J. B., Yanovich, R., Ketko, I., ... \& Reifman, J. (2018). Individualized estimation of human core body temperature using noninvasive measurements. Journal of Applied Physiology, 124(6), 1387-1402. doi: 10.1152/japplphysiol

[21] Bonidia, R. P., Rodrigues, L. A., Avila-Santos, A. P., Sanches, D. S., \& Brancher, J. D. (2018). Computational intelligence in sports: A systematic literature review. Advances in Human-Computer Interaction, 2018.

DOI: https://doi.org/10.1155/2018/3426178

[22] Morkūnas, M., Treigys, P., Bernatavičienè, J., Laurinavičius, A., \& Korvel, G. (2018). Machine learning based classification of colorectal cancer tumour tissue in whole-slide images. Informatica, 29(1), 75-90,

DOI:https://doi.org/10.15388/Informatica.2018.158

[23] Keshavarz Ghorabaee, M., Zavadskas, E. K., Olfat, L., \& Turskis, Z. (2015). Multi-criteria inventory classification using a new method of evaluation based on distance from average solution (EDAS). Informatica, 26(3), 435-451,

DOI: https://doi.org/10.15388/Informatica.2015.57

[24] Fujinawa, Y., \& Noda, Y. (2013). Japan's earthquake early warning system on 11 March 2011: performance, shortcomings, and changes. Earthquake Spectra, 29(1_suppl), 341-368. https://doi.org/10.1193/1.4000127

[25] Sparks, R., Carter, C., Graham, P., Muscatello, D., Churches, T., Kaldor, J., \& Ryan, L. (2010). Understanding sources of variation in syndromic surveillance for early warning of natural or intentional disease outbreaks. IIE Transactions, 42(9), 613-631.

DOI: $10.1080 / 07408170902942667$

[26] Sharma, A., \& Kumar, R. (2019). Computation of the reliable and quickest data path for healthcare services by using service-level agreements and energy constraints. Arabian Journal for Science and Engineering, 44(11), 9087-9104, DOI: https://doi.org/10.1007/s13369-019-03836-4

[27] Laxminarayan, S., Rakesh, V., Oyama, T., Kazman, J. B., Yanovich, R., Ketko, I., \& Reifman, J. (2018). Individualized estimation of human core body temperature using noninvasive measurements. Journal of Applied Physiology, 124(6), 1387-1402. doi: 10.1152/japplphysiol.

[28] Hepworth, P. J., Nefedov, A. V., Muchnik, I. B., \& Morgan, K. L. (2010). Early warning indicators for hock burn in broiler flocks. Avian Pathology, 39(5), 405-409. doi: 10.1136/vr.104066

[29] Keshavarz-Ghorabaee, M., Amiri, M., Zavadskas, E. K., Turskis, Z., \& Antucheviciene, J. (2018).
Simultaneous evaluation of criteria and alternatives (SECA) for multi-criteria decision-making. Informatica, 29(2), 265-280,

DOI: https://doi.org/10.15388/Informatica.2018.167

[30] Kumar, R., \& Saini, H. (2020). Secure, Resilient and Green Computing in Wireless Sensor Networks. Recent Advances in Electrical \& Electronic Engineering (Formerly Recent Patents on Electrical \& Electronic Engineering), 13(2), 128129. https://doi.org/10.1007/s12083-016-0511-y

[31] Sayago-Heredia, J., Pérez-Castillo, R., \& Piattini, M. (2021). A Systematic Mapping Study on Analysis of Code Repositories. Informatica, 1-42, DOI: https://doi.org/10.15388/21-INFOR454

[32] Sharma, A., \& Kumar, R. (2017). A framework for pre-computated multi-constrained quickest QoS path algorithm. Journal of Telecommunication, Electronic and Computer Engineering (JTEC), 9(36), 73-77. https://doi.org/10.1016/j.future.2017.09.010

[33] Leddy, J. J., Baker, J. G., Merchant, A., Picano, J., Gaile, D., Matuszak, J., \& Willer, B. (2015). Brain or strain? Symptoms alone do not distinguish physiologic concussion from cervical/vestibular injury. Clinical journal of sport medicine, 25(3), 237-242. doi: 10.1097/JSM.0000000000000128

[34] Sharma, A., \& Kumar, R. (2019). Risk-energy aware service level agreement assessment for computing quickest path in computer networks. International Journal of Reliability and Safety, 13(1-2), 96-124, DOI: https://doi.org/10.1504/IJRS.2019.097019

[35] Walter, S. D., Hart, L. E., McIntosh, J. M., \& Sutton, J. R. (1989). The Ontario cohort study of runningrelated injuries. Archives of internal medicine, 149(11), 2561-2564. https://doi.org/10.1001/archinte.

[36] Quaid, M. A. K., \& Jalal, A. (2020). Wearable sensors based human behavioral pattern recognition using statistical features and reweighted genetic algorithm. Multimedia Tools and Applications, 79(9), 6061-6083. https://doi.org/10.1007/s11042-019-08463-7.

[37] Akutsu, T., Miyano, S., \& Kuhara, S. (2000). Algorithms for identifying Boolean networks and related biological networks based on matrix multiplication and fingerprint function. Journal of computational biology, 7(3-4), 331-343. doi: $10.1089 / 106652700750050817$.

[38] Goodlin, G. T., Roos, A. K., Roos, T. R., Hawkins, C., Beache, S., Baur, S., \& Kim, S. K. (2015). Applying personal genetic data to injury risk assessment in athletes. PloS one, 10(4), e0122676. DOI:10.1371/journal.pone.0122676. 
